Uluslararası Mühendislik

Cilt/Volume:11 Sayı/Issue:1 Ocak/January 2019

Araştırma Makalesi / Research Article

\title{
Predicting the Turkish Stock Market BIST 30 Index using Deep Learning
}

\author{
Halil Raşo ${ }^{1}$ (D), Mehmet Demirci *1 (iD) \\ ${ }^{I}$ Gazi University, Faculty of Engineering, Department of Computer Engineering, Ankara, Turkey
}

Başvuru/Received: $21 / 05 / 2018$

Kabul/Accepted: $12 / 12 / 2018$

Son Versiyon/Final Version: $31 / 01 / 2019$

\begin{abstract}
The non-linearity and high change rates of stock market index prices make prediction a challenging problem for traders and data scientists. Data modeling and machine learning have been extensively utilized for proposing solutions to this difficult problem. In recent years, deep learning has proved itself in solving such complex problems. In this paper, we tackle the problem of forecasting the Turkish Stock Market BIST 30 index movements and prices. We propose a deep learning model fed with technical indicators and oscillators calculated from historical index price data. Experiments conducted by applying our model on a dataset gathered for a period of 27 months on www.investing.com demonstrate that our solution outperforms other similar proposals and attains good accuracy, achieving $0.0332,0.109,0.09,0.1069$ and 0.2581 as mean squared error in predicting BIST 30 index prices for the next five trading days. Based on these results, we argue that using deep neural networks is advisable for stock market index prediction.
\end{abstract}

\section{Key Words}

"Stock market index prediction; Deep learning; Deep Neural Network; Stock index; BIST" 


\section{INTRODUCTION}

Stock market prediction is a difficult task due to the huge amount of data to be processed, frequent and nonlinear stock price changes, and the diversity of influencing factors, such as national/global economic conditions and news, investors moods etc. In addition, the efficient market hypothesis states that stock movements are in accordance with the random walk model, thus making it highly improbable to predict their movement directions and prices.

Some investors use technical indicators and oscillators to build charts and patterns to help them discover price trends, and devise strategies for high-return investments. This method is called technical analysis and its proponents believe that the market discounts everything, prices move in trends and historical trends repeat themselves. Technical analysis or charting indicators focus on historical values and movements of index prices as the entry point of the financial market analysis, and build charts illustrating hidden points that investors can use in their investment strategies.

Another camp of investors rely on fundamental indicators rather than technical ones and they are called fundamental analysts. They analyze the volume of shares, financial and political news, investors' moods and other factors that influence the market. The main difference between these two schools of financial market analysis is the period of time that investment strategies consider. Technical analysis focuses on the next short time period whereas fundamental analysis considers longer time periods, i.e. at least one quarter or more.

Many traditional machine learning proposals like support vector machines, variant decision trees, k-nearest neighbors and artificial neural networks have been suggested for stock market prediction. These algorithms are powerful in many problems but in such highly volatile and non-linear problems they suffer from stability issues. Deep learning has proved itself a promising solution for such environments and showed good performance (Akita et al., 2016).

In this work, we propose a deep learning model trained on the most important technical oscillators of the BIST 30 Index of the Turkish stock market. These indicators are noted by famous technical analysts and www.investing.com, a popular website among traders and investors. To the best of our knowledge, this paper is the first in the literature using a deep learning model to predict Turkish stock market index prices. We have conducted our experiments on historical index data obtained from www.investing.com for a 27-month period from 01.01.2016 to 11.04.2018. Our model was able to produce good predictions with low error rates, as discussed in the evaluation section.

The rest of the paper is organized as follows. A brief review of related works is given in Section 2. Technical indicators and oscillators used in our proposal have been described and fundamental indicators are briefly mentioned in Section 3. Our proposal and the walkthrough of our method have been explained in detail in Section 4. Section 5 describes our experiments and presents the evaluation results. Finally, conclusions and future work areas are highlighted in Section 6.

\section{RELATED WORK}

Technical analysis and fundamental analysis are the two main schools of thought when it comes to analyzing the financial markets. While technical analysis focuses on historical data of stock prices and volumes, fundamental analysis gives significant weight to investors' sentiments, economic and political conditions and news. Nassirtoussi et al. (2015) proposed an approach to predict intraday forex currency-pair directions by analyzing breaking financial news headlines. Another work done by Shynkevich et al. (2015) analyzed the effects of market-related articles on stock trends and prices. The authors have developed a model to predict these values from industry-specific articles. Oliveir et al. (2017) analyzed the impact of microblogging data related to stock market news on the investors' sentiments. The authors forecast the returns, volatility and trading volume of diverse indices and portfolios from tweet messages. Ni et al. (2015) investigated the effects of investors' sentiments on stock market index prices and directions. According to their findings, the impact of investor sentiment is considerable for up to 2 years. Its effect is asymmetric, that is, it is positive and large for stocks with high returns in the short term, while negative and small in the long term. Leigh et al. (2002) studied the effectiveness of technical analysis approaches using multiple technical indicators and how they are used to achieve high return rates using decision making systems. They emphasized the importance of the "bull flag" price and volume pattern heuristic in getting abnormal results. Later, the indicators noted by these schools are used as inputs or features to prediction systems. Machine learning algorithms are the primary techniques used for predicting stock prices and directions. Gui et al. (2014) proposed an interesting approach through which the prediction is not a specific number but a limit instead. The authors transformed financial time series into fuzzy particle sequences and then used support vector machine to build a regression model on the lower and upper bounds to decrease the estimation error. Dechow et al. (2001) showed how short-sellers benefit from those factors in refining their investment strategies and maximizing their returns. Another work (Lewellen et al., 2010) emphasized the importance of key factors of fundamental analysis and suggested some improvements in empirical tests. Dechow et al. (2010) reviewed the various measures of "earning quality" and how it is related to the company fundamental performance. Qian et al. (2007) used the Hurst exponent to select highly predictable period, and later training patterns or indicators are generated by auto-mutual information and false nearest neighbor methods. Trained by an ensemble of inductive machine learning approaches such as artificial neural networks, decision trees and k-nearest neighbors, the model achieved 60- $65 \%$ of accuracy. 
Sands et al. (2015) compared different classification proposals: Support vector machine using least squares implementation, artificial neural networks, naïve Bayes classifier and SVM optimized by particle swarm optimization in building an investment portfolio with maximum gain and minimum risk. According to their experiments, SVM optimized by particle swarm optimization is capable of predicting the stock values with high accuracy. Another work (Ince et al., 2017) proposed a hybrid model for forecasting stock market movements. This model is composed of ICA for selecting important features between some technical indicators and then using kernel methods such as SVM, TWSVM, MPM, KFDA and random walk to build a model for predicting stock movements. According to their experiments on Dow-Jones, Nasdaq and S\&P500 indices, the models like ICA-SVM, ICATWSVM, ICA-MPM and ICA-KFDA have achieved high accuracy.

Bast1 et al. (2015) addressed the underpricing of Turkish companies in initial public offers traded in Istanbul Stock Exchange. They employed decision tree and support vector machine to investigate the key factors affecting the short-term performance of initial IPOs. Another approach (Chen et al., 2017) proposed a hybrid model composed of feature weighted of both support vector machine and k-nearest neighbors. The authors applied the model on two well-known Chinese stock market indices, Shanghai and Shenzhen stock exchange indices. Teixeira et al. (2010) combined technical analysis and k-nearest neighbors. Qian et al. (2007), Zhang et al. (2009), Moghaddam et al. (2016), and Boyacioglu et al. (2010) have investigated the use of artificial neural networks in stock market prediction. A recent work (Akita et al., 2016) using deep learning for stock market prediction was applied on Tokyo stock exchange market. The authors used paragraph vector to convert newspaper articles into distributed representations and used them with historical prices to predict values close to the actual stock prices.

We have noticed that most of the proposals based on technical analysis use their indicators heuristically without any features engineering or what recently technical analysts prefer to use between such indicators. For this reason, we did feature engineering and chose the most important ones noted by highly reputed global investment website. The closest study to our proposal was the one by Akita et al. (2016) due to its use of deep learning techniques but what differentiates our work is applying deep learning for Turkish stock exchange and using technical analysis in feature selection.

\section{BACKGROUND: TECHNICAL ANALYSIS AND INDICATORS}

In this section, we formalize the problem to be addressed. Prediction of stock index prices is a time series problem where each sample or observation contains the price values that an index can take during a trading day such as open, low, high, volume, trading date and closing price. The goal is to predict the price value for the following trading days with low errors. In this paper, we use samples and observations on a daily basis. This could be adapted by using other trading periods like minutes, weeks, months or even years. We express this in mathematical terms as follows:

$$
\text { closing }=f(\text { open, high, low, volume }, t)
$$

We build a deep learning model using certain technical analysis indicators as features. The following subsection describes technical analysis and the indicators we have used.

\subsection{Technical Analysis}

Technical analysts believe that historical prices of stock indices contain very important hidden information and they are highly related to the current prices. According to them, this could be explored by what they call indicators, oscillators and charts. So, the prices and directions of stock indices could be predicted by using such indicators. In that case we can rewrite the formula as given in Eq. 2.

$$
\text { closing }=f\left(\text { tech }_{\text {time-period }}\right)
$$

Technical analysis relies on analyzing certain indicators to extract information such as buy/sell signals from historical data and construct high-return investment strategies. There are approximately 150 technical indicators, but we only provide a brief description of the most important ones which are accepted by the popular investment portal www.investing.com.

\subsubsection{Relative Strength Index - RSI}

RSI is the most important momentum indicator developed by noted analyst J. Welles Wilder Jr and is explained in (Welles, 1978). It is used to identify overbought and oversold regions of the analyzed index. These regions are highly significant to the technical analyst or the trader to give buy or sell orders. What RSI does is observing the magnitude of recent gains and losses over a specified time period (14 trading days by default) to measure speed and change of price movements of an index. RSI is calculated by the following formula:

$$
R S I=100-\frac{100}{H H-L L}
$$

There are two important RSI levels: $(70,30)$. When the value of RSI exceeds 70 , this interpreted as a sell signal as the price becomes overvalued. On the other hand, when the RSI value falls under 30, a buy signal is generated. Some investors use extreme version of the RSI indicator where these two levels are $(80,20)$. It is important to mention that the time unit considered by the technical indicators in our calculations is one day but could be other trading units like minutes, hours, months or years. 


\subsubsection{Bollinger Bands - BB:}

BB is a momentum indicator or chart developed in the 1980s by noted trader John Bollinger (2001) through which the price of the index is bracketed by an upper and lower band along with a 21-day simple moving average (the default time period is 21 trading units). The upper and the lower band is double standard deviation of the middle band. According to Bollinger, when the price exceeds the upper band, it becomes overvalued and there will be a correction, so a sell opportunity is generated. Conversely, when it goes below the lower band, then the price is undervalued and it should be corrected, so a buy signal is generated.

\subsubsection{Stochastic Oscillator - STOCH:}

STOCH is a momentum indicator or oscillator frequently used by market traders and it compares the price of an index to the range of its prices over a certain period of time (The default time period is 14 trading units). The stochastic oscillator is calculated using the following formula:

Where:

$$
\% K=100 * \frac{\left(C-L_{14}\right)}{\left(H_{14}-L_{14}\right)}
$$

$C$ is the most recent closing price,

$L_{14}$ is the low of the 14 previous trading sessions,

$H_{14}$ is the highest price traded during the same 14-day period,

$\% K$ is the current market rate for the currency pair,

$\% D$ is 3-period moving average of $\% K$.

\subsubsection{Williams \% R:}

Williams \% R is a momentum indicator developed by famous technical analyst Larry Williams (1973), and it is the inverse of the Fast-Stochastic Oscillator. Williams \%R reflects the level of the closing price relative to the highest high for the look-back period. In contrast, the Stochastic Oscillator reflects the level of the closing price relative to the lowest low. Williams \%R is calculated by the following formula:

Where:

$$
\% R=\frac{H H-C}{H H-L L} *-100
$$

$H H$ is the highest high,

$C$ is the closing price,

$L L$ is the lowest low.

The time period considered by the formula is 14 trading units.

\subsubsection{Price Rate of Change - ROC:}

The price rate of change $(\mathrm{ROC})$ is a technical indicator of momentum that measures the percentage change in price between the current price and the price $\mathrm{n}$ periods in the past. It is calculated by using the following formula:

$$
R O C=\frac{C-C_{n}}{C_{n}} * 100
$$

Where

$C$ is the most recent closing price,

$C_{n}$ is the closing price n periods ago.

\subsubsection{Simple Moving Average - SMA:}

SMA is the simplest momentum indicator used by many traders and calculated by adding the closing price of the index for a number of time periods (The usual time period like other momentum indicators is 14 trading units) then dividing this total by the number of considered time periods as in the following formula:

\subsubsection{Exponential Moving Average - EMA:}

$$
S M A=\frac{\sum_{i=0}^{n} C}{n}
$$

An exponential moving average (EMA) is the exponential variation of the standard simple moving average except that in the former we give more importance to the latest closing prices of the index. This type of moving average reacts faster to recent price changes than a simple moving average and is calculated by using the following formula:

$$
\begin{gathered}
E M A_{t}=(C * K)+\left(E M A_{y} *(1-K)\right) \\
K=\frac{2}{(N+1)}
\end{gathered}
$$

Where:

$E M A_{t}$ is the current EMA value, $E M A_{y}$ is the previous EMA value, $N$ is the length of the EMA. 


\subsubsection{Commodity Channel Index - CCI:}

Another momentum indicator called Commodity Channel Index or CCI was developed by Donald Lambert and it measures the current price level relative to an average price level over a given period of time (14 trading units). CCI is relatively high when prices are far above their average, and CCI is relatively low when prices are far below their average. In this manner, CCI can be used to identify overbought and oversold levels which are important levels considered by traders to make buy and sell orders. It is calculated by using the following formula:

$$
C C I=\frac{\left(C-S M A_{20}\right)}{0.15 * \text { Mean deviation }}
$$

\subsubsection{On-Balance Volume - OBV:}

On-balance volume or OBV is a momentum indicator developed by Joseph E. Granville (1976) that considers index volume flow to predict changes in its price. According to him, the price of the index will eventually jump upward when volume increases sharply without a significant change in it and vice versa. It is computed by using the following formula:

$$
O B V_{c}=\sum V_{P N}
$$

Where:

$O B V_{C}$ in the current on-balance volume,

$V_{P N}$ is the positive-negative volume (volume is positive if current volume is bigger than previous volume)

\subsubsection{Moving average convergence divergence - MACD:}

Moving average convergence divergence or MACD is a trend-following momentum indicator that shows the relationship between two moving averages of prices. The MACD is calculated by subtracting the 26-day exponential moving average (EMA) from the 12-day EMA. A nine-day EMA of the MACD, called the "signal line", is then plotted on top of the MACD, functioning as a trigger for buy and sell signals. MACD is calculated by the following formula:

$$
M A C D=\left(E M A_{26}-E M A_{12}\right)
$$

\subsubsection{STOCHRSI:}

Some momentum indicators give a good performance when they are accompanied by other technical or momentum indicators. STOCHRSI is one such indicator used in technical analysis that ranges between zero and one. It is created by applying the Stochastic Oscillator formula to a set of Relative Strength Index (RSI) values rather than standard price data. Using RSI values within the stochastic formula gives traders an idea of whether the current RSI value is overbought or oversold - a measure that becomes specifically useful when the RSI value is confined between its signal levels of 20 and 80 .

\subsection{Fundamental Analysis:}

Fundamental analysts believe in fundamental factors rather than technical ones. They care about the intrinsic values of stocks and take into account everything related to the stocks such as earnings, market shares, financial conditions, news and investors' sentiments. Contrary to technical analysts, fundamental analysts perform their analysis and calculations for a sufficiently long time period and try to minimize their transactions. Eq. 13 gives the estimation from fundamental analysts' point of view.

$$
\text { closing }=f\left(\text { fund }_{\text {time-period }}\right)
$$

\section{Proposed Model}

Historical data of the stock index are quite simple and contain only few values that it can take during a trading unit (hour, day, month or year) such as open, high, low, volume and closing price. Our goal is to predict the closing price from these values, which is a challenging task due to the volatility of these prices. We need to calculate technical indicators from such values as these indicators hold valuable hidden information about prices. There are approximately 150 indicators, below we list the most important ones as noted by technical analysts and by a popular investment portal. Selecting and calculating these oscillators is the first step of our model which consists of two parts:

\subsection{Calculation of Technical Indicators:}

This step calculates technical indicators or oscillators from historical data of BIST 30 index price and volume. While some of these indicators depend only on the closing price of the index, others depend on the low and high as well as the closing price. For instance, one of the oscillators called On-Balance Volume (OBV) depends on the volume value of the index. The calculation of these oscillators is based on the default time period of each one. The output of this calculation will be the input of our deep NN. In other words, they will be accepted like its features.

\subsection{Deep Neural Network (Deep Learning):}

Artificial Neural Network or ANN is one of the most important research areas in artificial intelligence and machine learning. The main idea behind ANN is inspired by the natural neural network of the human nervous system. Neurons are imitated with computing units connected with each other in the form of a network through axons and dendrites. Each neuron or node receives 
inputs from other nodes through its dendrites, performs an operation on them and sends the result of that operation to other neurons. The inputs to the ANN (also known as features) are technical indicators in our case.

A perceptron is a binary classifier that uses a linear prediction function. Most ANNs are networks of perceptrons, also known as feed forward neural networks, organized into fully connected layers. While a perceptron is suitable when trying to build a linear decision boundary, simple ANN becomes unfeasible in the case of building a regression model with many features, hence deep neural networks are needed. Deep NNs are simply ANNs with more hidden layers and neurons in each of them as illustrated in Fig. 1. In the following subsection, we explain the steps taken in our work to build a regression model to predict the future prices of the BIST 30 index.

\section{Simple Neural Network}

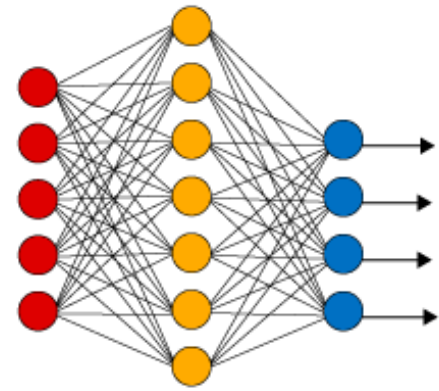

Input Layer

\section{Deep Learning Neural Network}

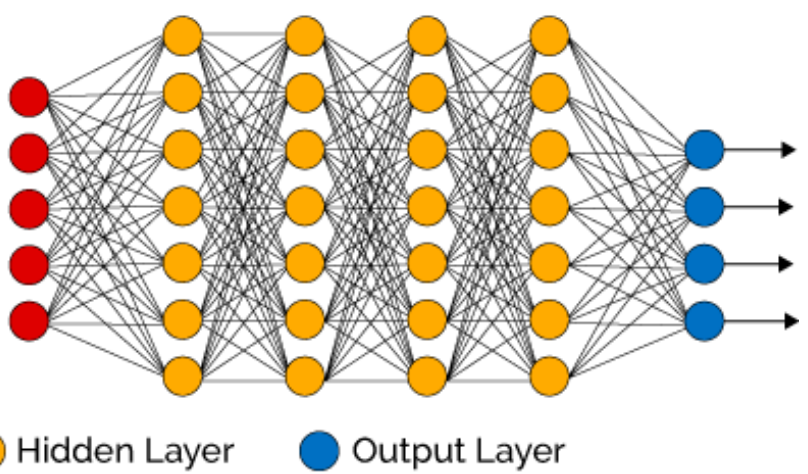

Fig. 1. General Structure of an artificial neural network. Deep neural networks consist of more hidden layers and more neurons in each hidden layer (Vázquez, F. 2017)

\subsection{Walkthrough}

Here, we provide a step-by-step explanation of the phases of our method.

\subsubsection{Gathering data:}

As our problem is analyzing the Turkish stock exchange market in order to predict future price movements of BIST 30, we needed to gather significant amount of financial data. One of the most reliable websites followed by many traders is

www.investing.com. We obtained our dataset from that website for a period of more than two years. The prices of stock indices are generally given in csv format containing the values of closing, opening, low, high, and volume of the index. Table 1 . shows a sample portion of the dataset.

Table 1. Sample of historical data of BIST 30

\begin{tabular}{llllll}
\hline Date & Price & Open & High & Low & Volume \\
\hline 04.01 .16 & $85,981.14$ & $87,428.49$ & $87,428.49$ & $85,023.80$ & 339.09 \\
05.01 .16 & $86,147.25$ & $85,981.14$ & $86,940.28$ & $84,502.58$ & 488.09 \\
06.01 .16 & $86,862.50$ & $86,147.25$ & $86,970.83$ & $84,904.24$ & 596.21 \\
07.01 .16 & $87,417.44$ & $86,862.50$ & $87,577.47$ & $84,994.29$ & 705.17 \\
08.01 .16 & $86,234.62$ & $87,417.44$ & $88,226.75$ & $85,932.68$ & 565.21 \\
11.01 .16 & $86,825.17$ & $85,933.88$ & $87,568.90$ & $85,517.25$ & 500.37 \\
12.01 .16 & $87,724.37$ & $86,783.72$ & $88,216.83$ & $86,094.12$ & 634.06 \\
\hline
\end{tabular}

\subsubsection{Data preparation:}

Cleaning and processing data is necessary in most cases before applying machine learning algorithms. The datasets related to financial markets suffer from several specific problems:

- Some companies may not exist any longer.

- The market is closed during national holidays and on the weekends.

- For technical problems prices contain negative errors.

These issues should be addressed when constructing a machine learning model. Two important preprocessing issues are normalization and finding correlated features. It is strongly advised to make the features data range [0, 1]. Fig. 2 and Fig. 3 show the histograms of the features before and after normalization. We see that all of the features are normalized, and their values are in the range $[0,1]$ except the price since it is not a feature, but the target value we are going to predict.

Another issue is finding out if, and which, features are correlated with each other. Such features should be eliminated as correlated features cause an ANN to overfit and have a bad impact on its performance. According to technical indicator formulas, we expect high correlation between SMA and EMA as they both represent moving averages. If so, we should eliminate one as it serves nothing. Fig. 4 confirms our intuition that these two features are highly correlated. It is important to notice that some indicators 
have more than one output and these outputs are correlated with each other we keep them as they are. Fig. 5 shows the pairwise features correlation. We see that OBV indicator is highly correlated with Bollinger bands (BB) indicator, so we drop it from our calculations. As a result, we have dropped two indicators (SMA and OBV), and used the remaining nine indicators from Section 3.1 in our model.

\subsubsection{Choosing a model:}

Selecting a suitable model is critical for the performance of machine learning. In this work, we try to predict stock index values, so we focus on regression. For reasons outlined in the introduction, we pick a deep neural network trained over technical oscillators obtained from technical indicators calculator.
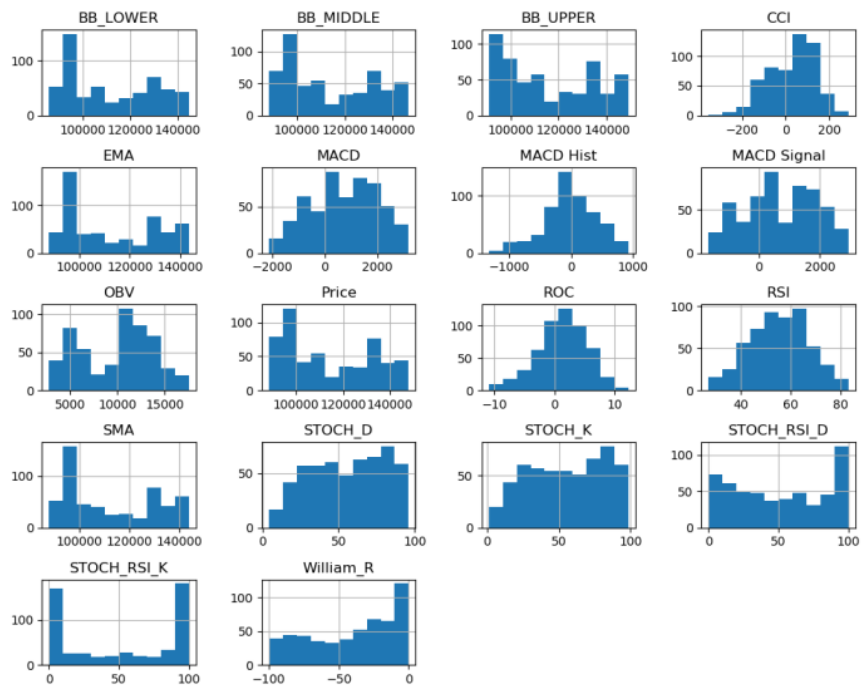

Fig. 2. Histogram of features before normalization
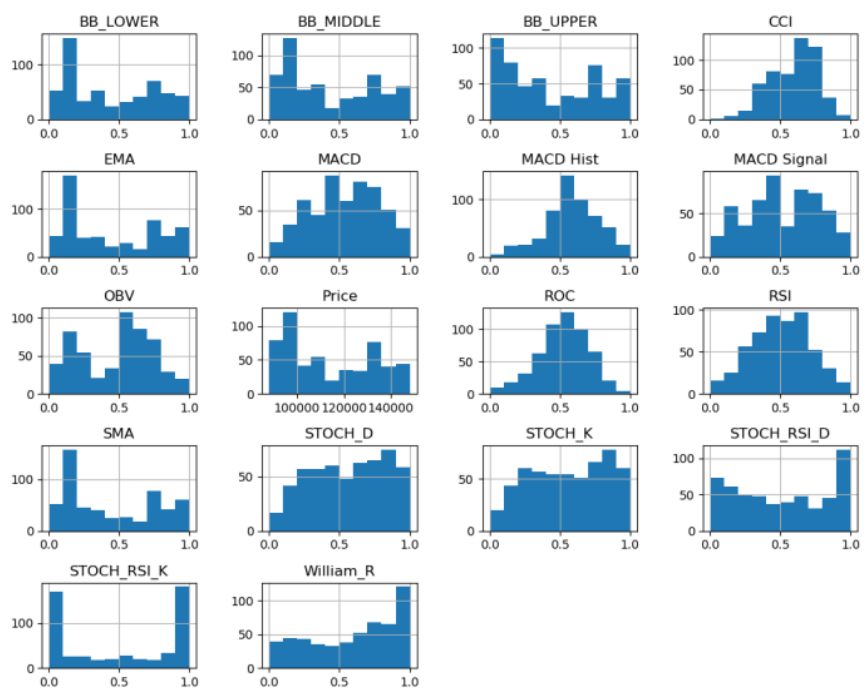

Fig. 3. Histogram of features after normalization

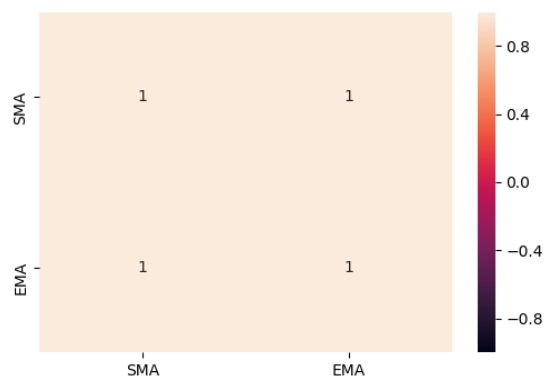

Fig. 4. High correlation between SMA and EMA 


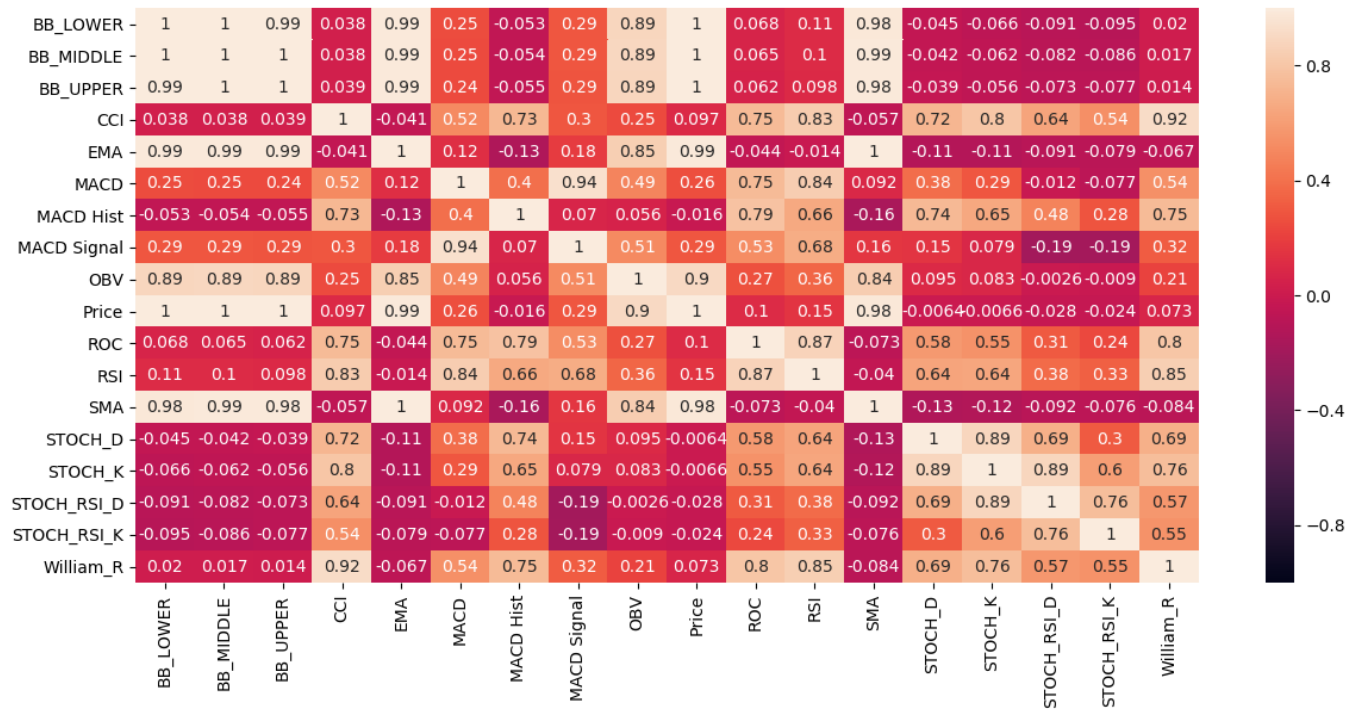

Fig. 5. Pairwise features correlations. OBV indicator is highly correlated with BB indicators.

\subsubsection{Training:}

Training a machine learning model means adjusting the model parameters to reduce the loss and achieve the desired prediction. Parameters in our case are neuron weights and bias. We train our deep NN using Keras API over the TensorFlow framework developed by Google. We have used Keras sequential model API with ReLU (Rectified Linear Unit) Activation Function for hidden layers. As our target model is a regression model, there is no need for an activation function in the output layer. The last point that we should mention is the optimizer algorithm which is responsible for adjusting weights and bias. We have used Adam adaptive moment estimation optimizer.

\subsubsection{Evaluation:}

A common split ratio between the training set and the test set is $80-20$, and we use this ratio. We could not use cross validation when splitting the test set from the training set because our problem is time-series prediction and in such a situation the algorithm learns on the first portion of the dataset (training set) and is then evaluated on the test set (the last portion of the data). In other words, the algorithm could not be trained on recent data and tested on older data. So, our model is trained on the first $80 \%$ of our dataset (BIST 30's historical data for 27 months of trading) and it is tested over the last $20 \%$ after shifting the y values according to the target trading day.

\subsubsection{Hyperparameter tuning:}

Typically, it is hard to generate a robust and highly accurate model on the first run of an algorithm. Thus, some parameters of the model should be readjusted to decrease the loss of the regression model. Possible changes include increasing or decreasing the number of hidden layers, number of the neurons in such layers, activation function and optimizer algorithm used for training the network. We have achieved the desired performance with 7 hidden layers and 2 dropout layers, Relu as an activation function and Adam as an optimizer. Fig. 6. shows our network where the input layer has 15 neurons (technical indicators after dropping SMA and $\mathrm{OBV}$ ) and the output layer has only one neuron as we try to predict the index value (one value). Additionally, there are 7 hidden layers with 512, 256, 128, 64, 32, 16, and 8 neurons. There are two dropout layers after the first and the second hidden layer with $30 \%$ and $25 \%$ as dropout rates respectively. 


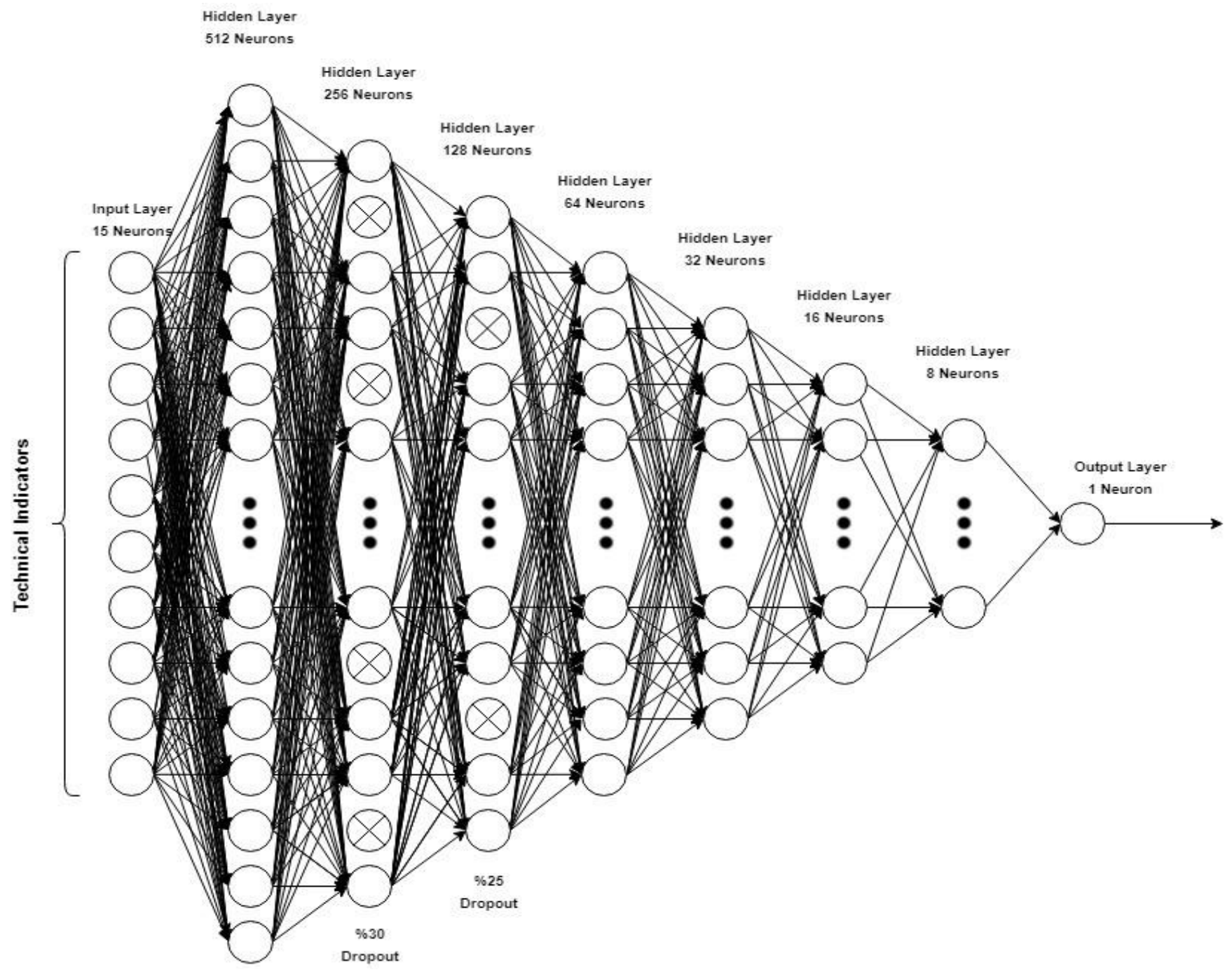

Fig. 6. Deep neural network used in our work

\subsubsection{Prediction:}

After adjusting the parameters which helped to obtain an acceptable model, this model applied over the test set data to make predictions and evaluate the performance using various metrics.

\section{EXPERIMENTAL RESULTS}

We have conducted our experiments on a dataset gathered from 01.01.2016 to 11.04.2018 on www.investing.com. Each observation or row contains the trading date, closing, opening, low and high price values as well as the volume and change percentage with respect to the previous trading day. After preprocessing our data and clearing out negative and null values, we calculate the technical indicators or oscillators to be used as features in our model. We split the dataset into training set X and test set y and train the deep neural network. We use $80 / 20$ as the training/test split ratio where the first $80 \%$ of data (BIST's historical data for 27 months of trading) is used as training set and the last $20 \%$ of data is used as test set. The y values in each training and test portion are shifted according to the trading day. For example, if we want to predict the index value for the next trading day we shift the y values with one and for second trading day with two and so on.

One important point we should mention here is that as our problem is a time-series problem, in order to predict the price value after one or two trading days we should shift the target column as much as needed. For example, to predict the index closing price for the next day, we should shift y by one row, and by two rows in the case of predicting the price for the next two days. This mechanism is known as window mechanism.

As our problem is a regression problem, we use mean squared error (MSE), R2 score, mean absolute error (MAE) and mean absolute percentage error (MAPE) metrics to evaluate the performance of our model and compare it with other methods in the literature (Patel et al., 2015), (Sakarya et al., 2015). We have used multiple performance metrics as each metric yields some valuable information not supplied by the others. For example, sometimes the MSE is very low but the R2 score is negative, which means that the model is arbitrary and did not train well. Generally, the metrics except R2 are considered better when close to zero, whereas the best value for $\mathrm{R} 2$ is 1 . 
Fig. 7. shows the loss achieved by our model. As the loss trends towards and stays close to 0, this means that our model is trained well. Table 4 gives the performance metrics achieved by our deep learning model for the first five trading days and compare them with two other techniques: SVR (support vector regression) and regular ANN (artificial neural network). Our deep learning model clearly outperforms ANN for the first five trading days and SVR for the first four trading days, whereas SVR gives better results for the fifth trading day. Also, our deep model outperforms the proposals presented by Patel et al. (2015) and by Sakarya et al. (2015) as illustrated in Table 2 and Table 3 using the metrics reported in those works. Fig. 8. plots predicted closing prices vs. real closing prices for the five next trading days. We observe that the predicted prices closely follow the actual trends.

Table 2. Comparison between our proposal and work by Patel et al. (2015) using MSE as metric.

\begin{tabular}{lll}
\hline Trading day & Our Proposal & Other proposal \\
\hline 1st trading day & 0.0332 & 0.4427 \\
2nd trading day & 0.1090 & 0.8748 \\
3rd trading day & 0.0900 & 1.3556 \\
4th trading day & 0.1069 & 1.8445 \\
5th trading day & 0.2581 & 2.3455 \\
\hline
\end{tabular}

Table 3. Comparison between our proposal and work by Sakarya et al. (2015) using MAPE as metric.

\begin{tabular}{lll}
\hline & Our Proposal & Other proposal \\
\hline next trading day & 1.0676 & 2.015 \\
\hline
\end{tabular}

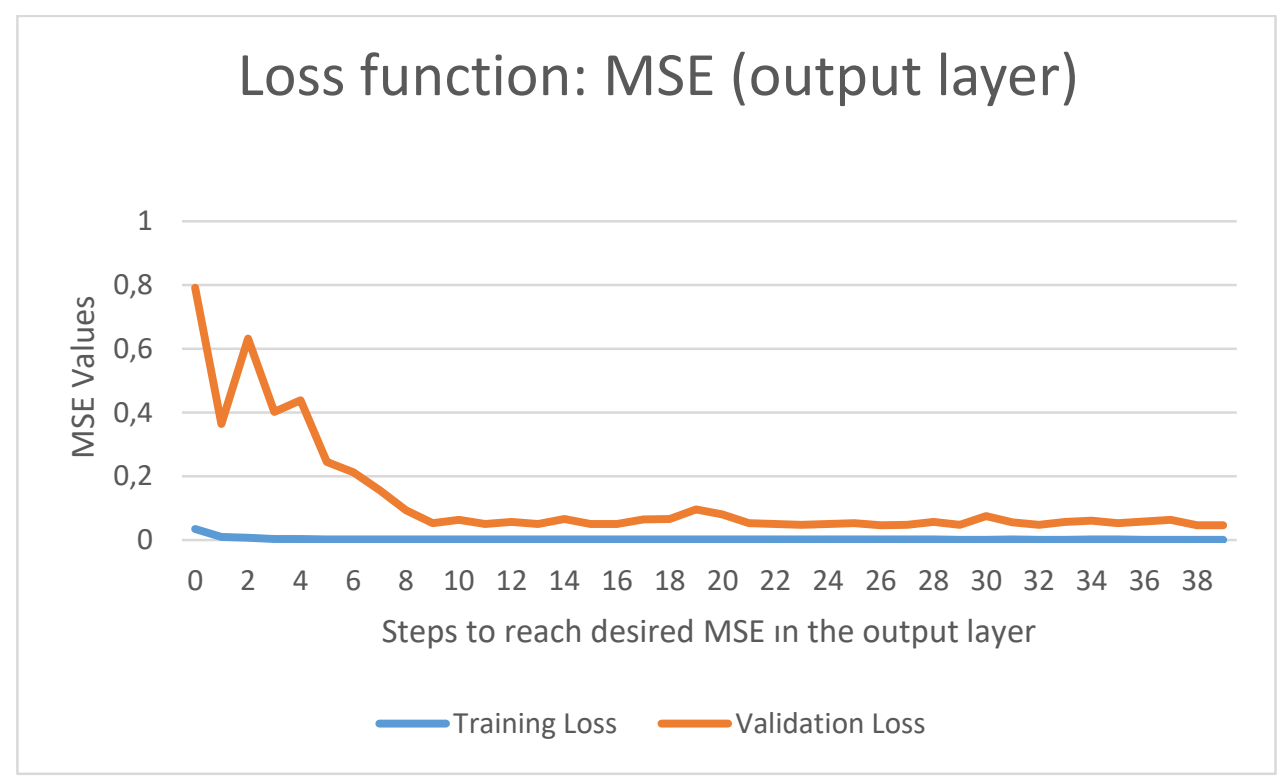

Fig. 7. Loss achieved by our model for the next trading day 


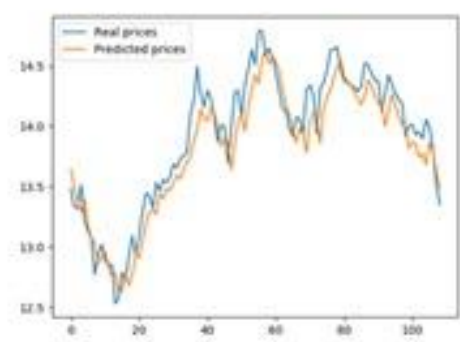

Fig. 8.a Real vs predicted prices for 1st trading day.

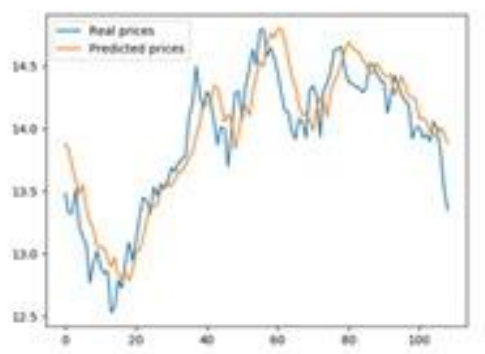

Fig. 8.b Real vs predicted prices for 2 nd trading day.

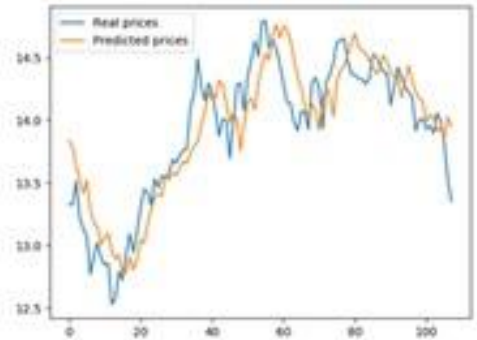

Fig. 8.c Real vs predicted prices for 3rd trading day.

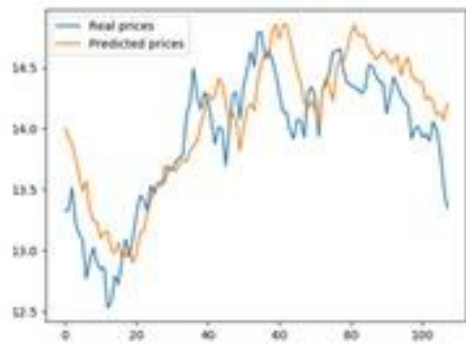

Fig. 8.d Real vs predicted prices for 4 th trading day.

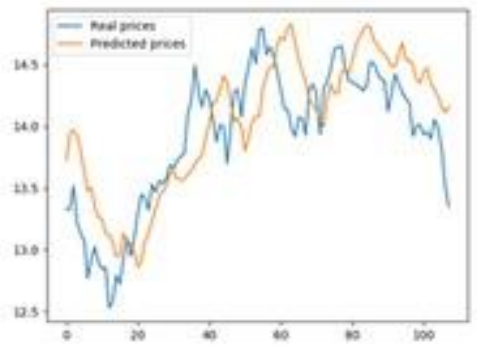

Fig. 8.e Real vs predicted prices for 5 th trading day.

Fig. 8. Actual vs. predicted prices for the next five trading days.

Table 4. Performance comparison for the next five trading days.

\begin{tabular}{|c|c|c|c|c|c|}
\hline & & MSE & R2 Score & MAE & MAPE \\
\hline \multirow[t]{3}{*}{$1^{\text {st }}$ Trading Day } & Deep Model & 0.0332 & 0.8937 & 0.1487 & 1.0676 \\
\hline & SVR & 0.1846 & 0.410 & 0.3823 & 2.7185 \\
\hline & ANN & 0.8866 & -1.8311 & 0.7703 & 5.537 \\
\hline \multirow[t]{3}{*}{ 2nd Trading Day } & Deep Model & 0.1090 & 0.6518 & 0.2714 & 1.6726 \\
\hline & SVR & 0.1448 & 0.5374 & 0.3188 & 2.2634 \\
\hline & ANN & 0.6971 & -1.2260 & 0.6747 & 4.8565 \\
\hline \multirow[t]{3}{*}{ 3rd Trading Day } & Deep Model & 0.0900 & 0.7135 & 0.2532 & 1.6052 \\
\hline & SVR & 0.1009 & 0.6789 & 0.2580 & 1.8394 \\
\hline & ANN & 0.4554 & -0.4488 & 0.5635 & 4.0630 \\
\hline \multirow[t]{3}{*}{ 4th Trading Day } & Deep Model & 0.1069 & 0.6598 & 0.2669 & 1.6103 \\
\hline & SVR & 0.0996 & 0.6831 & 0.2609 & 1.8694 \\
\hline & ANN & 5.9874 & -18.045 & 2.2313 & 15.8569 \\
\hline \multirow[t]{3}{*}{ 5th Trading Day } & Deep Model & 0.2581 & 0.1787 & 0.4115 & 3.1046 \\
\hline & SVR & 0.1496 & 0.5240 & 0.3160 & 2.2551 \\
\hline & ANN & 2.9615 & -8.4205 & 1.6230 & 11.5143 \\
\hline
\end{tabular}

\section{CONCLUSIONS AND FUTURE WORK}

The non-linearity and high volatility of stock market index prices make it challenging to forecast these prices. Successful prediction of stock market index values would immensely help investors devise a high-return investment strategy. Generally, stock market prediction can be categorized into two camps in terms of the features used to build prediction models: Technical analysis-based 
proposals, fundamental analysis-based proposals. We addressed the BIST 30 index prediction problem using deep learning where features are selected from common important technical indicators. Using data from 01.01.2016 to 11.04.2018, we trained and tested our model to show that our model outperforms other techniques like ANN and SVR as well as comparable proposals in the literature (Patel et al., 2015, Sakarya et al., 2015). Therefore, we conclude that deep learning in this context has proved itself as a promising solution for such a complex task.

Stock market index prediction can be divided into two main broad categories in terms the output of predictions: Stock index price prediction (regression model, which is what we have focused on) and stock index direction prediction (classification model) which can be either up or down. The latter is important for building investment strategies containing more than one index. In future work, we plan to predict the index direction using deep learning with the same indicators. Another future work area is combining fundamental and technical indicators and using them together as features of the deep neural network. Also, another potential work could be adding breaking news to features sets to make features more complete and improve learning performance. Finally, all proposals are currently applied on offline datasets, and it would be useful to extend the model to handle live data as well.

\section{REFERENCES}

Akita, R., Yoshihara, A., Matsubara, T. \& Uehara, K. (2016). Deep learning for stock prediction using numerical and textual information. IEEE, Computer and Information Science (ICIS).

Bast1, E., Kuzey, C. \& Delen, D. (2015). Analyzing initial public offerings' short-term performance using decision trees and SVMs, $73,15-27$.

Bollinger, J. (2001). Bollinger on Bollinger Bands, McGraw-Hill Education.

Boyacioglu, M. A. \& Avci, D. (2010). An Adaptive Network-Based Fuzzy Inference System (ANFIS) for the prediction of stock market return: The case of the Istanbul Stock Exchange. Elsevier, Expert Systems with Applications, 37(12), 7908-7912.

Chen, Y. \& Hao, Y. (2017). A feature weighted support vector machine and K-nearest neighbor algorithm for stock market indices prediction. Elsevier, Expert Systems with Applications, 80, 340-355.

Dechow, P., Ge, W. \& Schrand, C. (2010). Understanding earnings quality: A review of the proxies, their determinants and their consequences. Elsevier, Journal of Accounting and Economics, 50(2-3), 344-40.

Dechow, P. M., Hutton, A. P., Meulbroek, L. \& Sloan, R. G. (2001). Short-sellers, fundamental analysis, and stock returns. Elsevier, Journal of Financial Economics, 61(1), 77-106.

Gui, B., Wei, X., Shen, Q. Qi, J. \& Guo, L. (2014). Financial Time Series Forecasting Using Support Vector Machine. IEEE, CIS, 15-16.

Ince, H. \& Trafalis, T. B. (2017). A Hybrid forecasting model for stock market prediction. Economic Computation and Economic Cybernetics Studies and Research, 51(3).

Leigh, W., Purvis, R. \& Ragusa, J. M. (2002). Forecasting the NYSE Composite Index with Technical Analysis, Pattern Recognizer, Neural Network, and Genetic Algorithm: A Case Study in Romantic Decision Support. Elsevier, Decision Support Systems, 32, 361-377.

Lewellen, J. (2010). Accounting anomalies and fundamental analysis: An alternative view. Elsevier, Journal of Accounting and Economics, 50(2-3), 455-466.

Moghaddam, A. H., Moghaddam, M. H. \& Esfandyari, M. (2016). Stock market index prediction using artificial neural network. Elsevier, Journal of Economics, Finance and Administrative Science, 21(41), 89-93.

Nassirtoussi, A. K., Aghabozorgi, S., Wah, T. Y. \& Ngo, D. C. L. (2015). Text mining of news-headlines for FOREX market prediction: A Multi-layer Dimension Reduction Algorithm with semantics and sentiment. Elsevier, Expert Systems with Applications, 42(1), 306-324.

Ni, Z., Wang, D. \& Xue, W. (2015). Investor sentiment and its nonlinear effect on stock returns-New evidence from the Chinese stock market based on panel quantile regression model. Elsevier, Economic Modelling, 50, 266-274.

Oliveir, N., Cortez, P. \& Areal, N. (2017). The impact of microblogging data for stock market prediction: Using Twitter to predict returns, volatility, trading volume and survey sentiment indices. Elsevier, Expert Systems with Applications, 73, $125-144$.

Patel, J., Shah, S., Thakkar, P. \& Kotecha, K. (2015). Predicting stock market index using fusion of machine learning techniques. Elsevier, Expert Systems with Applications, 42, 2162-2172. 
Qian, B. \& Rasheed, K. (2007). Stock market prediction with multiple classifiers. Springer, Applied Intelligence, 26(1), 25-33.

Sakarya, Ş., Yavuz, M., Karaoğlan, A. D. \& Özdemir, N. (2015). Stock market index prediction with neural network during financial crises: A review on Bist-100. 1, 2, 53-67.

Sands, T. M., Tayal, D., Morris, M. E. \& Monteiro, S. T. (2015). Robust stock value prediction using support vector machines with particle swarm optimization. IEEE, Evolutionary Computation (CEC).

Shynkevich, Y., McGinnity, T.M., Coleman, S. \& Belatreche, A. (2015). Stock price prediction based on stock-specific and subindustry-specific news articles. IEEE, Neural Networks (IJCNN), 12-17.

Teixeira, L. A. \& De Oliveira, A. L. I. (2010). A method for automatic stock trading combining technical analysis and nearest neighbor classification. Elsevier, Expert Systems with Applications, 37(10), 6885-6890.

Vázquez, F. (2017). Deep Learning made easy with Deep Cognition. https://becominghuman.ai/deep-learning-made-easy-withdeep-cognition-403fbe445351.

Welles Jr, J. (1978). New Concepts in Technical Trading Systems, Hunter Publishing Company, Greensboro, NC.

Williams, L. (1973). How I Made One Million Dollars Last Year Trading Commodities, Conceptual Management, Montery, CA. Joseph E. Granville (1976). Granville's New Strategy of Daily Stock Market Timing for Maximum Profit, Prentice-Hall, Inc., ISBN 0-13-363432-9.

Zhang, Y. \& Wu, L. (2009). Stock market prediction of S\&P 500 via combination of improved BCO approach and BP neural network. Elsevier, Expert Systems with Applications, 36(5), 8849-8854. 